Le cor et la cotte : le corps à l'épreuve de la fidélité dans le Roman de Tristan en prose et dans Guillaume d'Angleterre

Christine Ferlampin-Acher (CELAM-CETM)

Guillaume d'Angleterre, roman en vers dont l'attribution à Chrétien de Troyes, souvent discutée, me paraît très peu sûre, ${ }^{1}$ et le Roman de Tristan en prose n'ont en apparence guère de points communs : d'un côté la reprise romanesque de la trame hagiographique de la vie de saint Eustache, de l'autre un vaste roman amplifiant l'histoire des deux amants de Cornouailles. Cependant ces deux textes présentent en commun une utilisation intéressante du cor, en relation d'une part avec l'histoire d'un couple marié, Marc et Iseult dans un cas, Guillaume et Gratienne de l'autre, et d'autre part avec une cotte coupée, tailladée, celle du Chevalier à la Cotte Maltaillie dans le texte en prose, et celle que Guillaume dans le roman en vers partage en deux, sur le modèle de saint Martin ou de saint Gilles, et qui sert à envelopper ses deux fils nouveau-nés. La ressemblance n'est-elle que superficielle? Ou bien peut-on mettre en évidence une configuration d'objets signifiante ? Il me semble, et c'est ce que je vais tenter de montrer, que dans les deux cas le texte travaille à partir du cor qui met la fidélité à l'épreuve, tel qu'on le trouve dans le Lai du Cor de Robert Biket, et que la conjointure entre le cor et la cotte est signifiante, même si dans un cas il s'agit d'une corne à boire et dans l'autre d'un cor de chasse. La reprise du lai du Cor est évidente dans le Tristan en prose, et passe par l'insertion d'un récit bref dans la conjointure complexe du roman ${ }^{2}$ : si elle rejoue, comme l'a montré Nathalie Koble, l'épisode du boivre amoureux et renvoie à un Graal désacralisé ${ }^{3}$, cette scène me paraît aussi contribuer à structurer fortement le roman, dans la mesure où elle trouve un répondant dans la figure de Brunor, le Chevalier à la Cotte

\footnotetext{
${ }^{1}$ Les références de Guillaume d'Angleterre seront données à partir de: C. Ferlampin-Acher, Guillaume d'Angleterre, édition et traduction, Paris, Champion Classiques, 2007. Pour le Tristan en prose, on se référera à l'analyse de E. Löseth, Le Roman en prose de Tristan, le Roman de Palamède et la Compilation de Rusticien de Pise, analyse critique d'après les manuscrits de Paris, Paris, Bouillon, 1891, réimpr. New York, Burt Franklin, 1972. Les éditions consultées sont : Le roman de Tristan en prose, éd. R.-L. Curtis, t. II, Leyden, E. J. Brill, 1976 ; Le roman de Tristan en prose, éd. sous la direction de Ph. Ménard, Genève, Droz, 1987-1995, t. IX, éd. L. Harf-Lancner, 1997 et Le roman de Tristan en prose, version du manuscrit BnF. fr. 757, Paris, Champion, t. V, éd. C. Ferlampin-Acher, 2007.

${ }^{2}$ Voir Beate Schmolke-Hasselmann, «L'intégration de quelques récits brefs arthuriens (Cor, Mantel, Espee) dans les romans arthuriens du XIIIe siècle », dans Le récit bref au Moyen Âge, actes du colloque d'Amiens (mai 1979), éd. D. Buschinger, Paris, Champion, 1979, p. 57-72.

${ }^{3}$ Voir dans ce même volume,
} 
Maltaillie, un double de Tristan, sur lequel se clôt le roman. Dans Guillaume d'Angleterre, si la reprise du lai du cor n'est en rien explicite mais peut se dégager d'une lecture en profondeur, la conjonction avec la cotte est obvie, puisque le cor et la cotte sont les deux attributs nobiliaires principaux dont se dépouille successivement le roi.

L'étude de ces deux textes mettra en évidence que la conjonction entre les deux objets sert une réflexion sur la fidélité, le désir, la mise à l'épreuve du corps et son masquage.

\section{Le Tristan en prose}

\section{a. L’épisode du cor dans le Tristan en prose}

Dans le Tristan en prose, l'épreuve du cor a lieu juste avant l'épisode des faux, grâce auquel Andret tente de dénoncer les deux amants ( $\$ 48$ de l'analyse d'E. Löseth) et attise la haine de Marc. Or l'épreuve du cor est détournée, Marc et ses barons étouffant l'affaire, avant que les faux peu après ne rendent plus difficile l'aveuglement royal. L'épisode du cor est donné dans le tome II de l'édition Curtis, §526-531. Lamorat, l'un des fils de Pellinor de Listenois, rencontre près d'une fontaine une demoiselle, deux écuyers et un chevalier : $L a$ demoisele portoit a son col un cor d'ivoire le plus bel et le plus riche qui pieça eüst esté veüz (p. 129). Lamorat demande des nouvelles de la cour et des aventures. Le chevalier lui répond au sujet du cor qui sera l'objet d' « une des plus beles aventures que vos onques veïssiéz [...]. Et s'il est biax, encores est il plus merveilleux, car nule dame qui ait geüe avec autre que avec son mari n'i puet boire que li vins ne s'espande sor lui. Mes se dame puet estre trovee qui n'ait vers son mari mesfait ensi com je vos ai dit, seürement i puet boire que li vins ne respendra sor li » ( p. 129).

Lamorat s'effraie : "Et s'il avenoit que madame la roïne Genevre, qui est ores la plus haute dame dou monde, se fust joee par aventure a aucun chevalier de son ostel, si covendroit que li rois en seüst la verité por l'espreve de cesti cor» (p. 129).

Le chevalier refusant de dire à Lamorat qui envoie le cor, un combat a lieu. Vaincu, il est contraint de renseigner de Lamorat: le cor a été envoyé par Morgain, qui hait Arthur. Lamorat en déduit que ceste merveille de cest cor a ele ensi establi par son enchantement por metre mortel haine entre le roi Artus et la roüne Genevre, et il ordonne au chevalier de porter le cor à la cour de Marc : « et bien sache Tristanz que por haine et por male volenté de li est cil cors envoiez en Cornoaille » (p. 130). 
Lamorat opère un détournement : l'épreuve du cor, qui chez Robert Bicket, a lieu à la cour d'Arthur, est délocalisée à la cour de Marc. Dans ce transfert se lit la rivalité entre deux univers romanesques, rivalité qui est à la base du Tristan en prose, second venu après le Lancelot, et qui passe en particulier par la concurrence entre les deux héros ${ }^{4}$. Cependant ce détournement ne correspond pas à une falsification de l'objet, mais au contraire il semble permettre l'accomplissement de la véritable mission de celui-ci. Le discours de Lamorat insiste en effet plus sur le mot Cornoailles (repris quatre fois) que sur les noms de Marc, Iseult et Tristan : li chevaliers qui point ne l'aime li envoie cest cor por esprover la bonté des dames de Cornoailles [...] ; ; por male volenté de li est cil cors envoiez en Cornoaille (p. 130). Si la langue associe cor et Cornouailles, c'est que la vérité de celui-ci s'y trouve.

A la cour, Marc est impatient de tester l'objet. Tristan, réticent, demande qui envoie le cor, et à la réponse du chevalier, devine qu'il s'agit de Lamorat, ce qui le mécontente. Marc, heureux de contrarier Tristan, se hâte d'accomplir l'épreuve. Iseult tente de différer celle-ci, au prétexte que le cor est un enchantement, venu de Grant Bretaigne por correcier les hautes dames qui n'ont mie fait a la volenté de toz les enchanteors ne de totes les enchanteresses de la Grant Bretaigne (p. 132). Outre sa clairvoyance (c'est effectivement Morgue qui envoie le cor), il est à noter qu'Iseult rappelle discrètement la naissance d'Arthur : elle revient au péché originel, à Merlin, à Morgue, voire à Viviane, et en même temps discrédite le merveilleux. Iseult doit néanmoins se soumettre à l'épreuve et échoue : et quant ele cuide boevre, li vins espant sor li une grant partie (p. 132). Le roi s'exclame: «Bien vos iestes provee » et la condamne à mort, pour trahison. Mais Iseult conteste la validité de la «preuve »: l'enchantement ne vaut pas. A nouveau, le merveilleux est discrédité. Cette double mise en cause des enchantements et des enchanteurs, insistant sur leur impuissance et leur dévalorisation, est significative : par ce biais, c'est le philtre qui est nié dans sa vertu magique. L'épreuve signale donc la culpabilité d'Iseult doublement: la reine a fauté avec un autre homme (c'est ce que dit l'épreuve elle-même); qui plus est, elle est responsable (et non le philtre, puisque le merveilleux est discrédité : c'est Iseult elle-même qui involontairement

\footnotetext{
${ }^{4}$ Cette concurrence a été analysée par Adeline Richard dans la thèse qu'elle a consacrée à la comparaison des couples Lancelot Guenièvre et Tristan Yseult dans les deux romans en prose dont ils sont les héros, Amour et passe amour, Lancelot-Guenièvre, Tristan-Yseut dans le Lancelot en prose et le Tristan en prose. Etude comparative, thèse soutenue sous la direction de Ch. Connochie-Bourgne, à l'université d'Aix-en-Provence, 2003.

${ }^{5}$ On notera la récurrence des sons or et l'écho cor / Cornoailles.
} 
nous le dit). Le cor signale donc la culpabilité. A nouveau la proximité phonétique des mots, associant cor et corpe (coulpe), parle: ceste dame en est corpable, se corpe i avient (p. 133).

Pourtant l'affaire est étouffée. Toutes les dames sauf quatre ayant échoué, les hommes du roi refusent de punir leurs femmes: «por ce se vos volez a la roïne mal, nel volons nos pas a nos moilliers » (p. 133). Le nom des quatre preudefemmes ne sera même pas donné : on est bien loin de la première Continuation de Perceval où l'épreuve du cor aboutit à la mise en valeur de la fidélité de Guignier ${ }^{6}$. Finalement, li rois est auques liez de ceste novele, car tot deïst il ce por chastier la roïne, ne vosist il pas sa mort, s'il ne veïst plus apertement son mesfait qu'il ne l'avoit veüe. Il décide de tenir ceste chose a fable, à mensonge car il y trouvera plus d'honneur (p. 133). Le roi n'a donc pas cru le signe : celui-ci est en attente d'une confirmation qui viendra avec l'épreuve des faux Le vin répandu n'est qu'une métaphore du sang : avec les faux, il s'agira de sang véritable, plus parlant : l'épreuve renvoie à la perte de la virginité. Cette reprise de l'épreuve du cor dans le Tristan en prose est une façon d'en finir avec l'ancien monde d'Arthur et de passer de Lancelot, placé sous le signe de la culpabilité, e à Tristan. Par ailleurs on a l'impression que cette aventure n'est qu'une étape dans le processus qui conduit le roi à la connaissance. Le cor ne serait qu'un épisode au sens étymologique du terme (qui désigne la partie du drame entre deux entrées du chœur dans le théâtre grec, et corrélativement, un élément accessoire). L'épreuve du cor serait anecdotique et secondaire : reléguée dans un temps révolu, celui de Lancelot, et appelée à être dépassée par l'épreuve des faux, plus authentique et plus visible, plus littérale et moins littéraire, on pourrait la supprimer sans danger.

Plusieurs indices invitent à douter de cette hypothèse. D'une part, l'épisode a semblé important : il a été souvent illustré ${ }^{7}$. De plus dans cet épisode le problème posé par l'épreuve du cor, la culpabilité d'Iseult, est à la fois amplifié par le fait que la reine rejette la lecture merveilleuse du monde, et escamoté de façon trop voyante pour être anecdotique. Par ailleurs l'épisode fonctionne en système avec la figure du Chevalier à la Cotte Maltailliee, qui rejoue l'épreuve de fidélité, à partir, c'est mon hypothèse, du modèle du Cort Mantel, et ce jusqu'à l'extrême fin du roman : l'épreuve du cor n'est pas une conclusion ; au contraire c'est une tête de pont à partir de laquelle se développe le roman, jusque dans ses derniers rebondissements, en relation avec Brunor, le Chevalier à la Cotte Maltailliee.

\footnotetext{
${ }^{6}$ Ed. W. Roach, Paris, Le Livre de Poche, Lettres Gothiques, 1993, v. 3106.

${ }^{7}$ Retenons par exemple les miniatures BnF. fr. 102, f. 67v, BnF. fr. 100, f. 95v, BnF. fr. 97, f. 59, toutes du XVe siècle.
} 
Ce chevalier et Tristan aiment tous deux Iseult et leur rivalité est marquée par le fait que pendant longtemps ils ne seront jamais présents ensemble à la Joyeuse Garde. De même qu'Iseult à la cour de Marc échoue à l'épreuve du cor, de même Brunor porte une cotte maltaillie où se lirait l'infidélité de sa dame. Brunor est à la fois le porte-parole de l'amour courtois parfait, puisqu'il reste inébranlable dans son amour et l'homme-sandwich affichant aux yeux du monde entier la faute d'Iseult. Dans ce cheminement auquel je vous convie, on passe de l'épreuve du cor, obvie, à une exploration souterraine, nettement plus incertaine, de la cotte maltaillie. L'interprétation que je propose n'est contredite par aucun élément du texte, elle peut expliquer l'importance que le récit accorde à ce personnage étrange qu'est Brunor, et elle éclaire la fin du roman : autant de caractéristiques qui justifient son examen. Pour passer du cor à la cotte, il est nécessaire d'examiner d'abord comment se constitue la figure du Chevalier à la Cotte Maltaillie.

\section{b. Brunor}

Le personnage de Brunor s'élabore progressivement ${ }^{8}$ : il ressemble d'abord à Lancelot, puis à Perceval, et finalement, et c'est en tant que double de Tristan qu'il s'impose. Il apparaît dans le roman loin en amont, un peu après l'épisode du cor ${ }^{9}$, lors d'une cour, à Pâques, après que Lamorat a donné diverses nouvelles (Vienne 2542, f. $43 \mathrm{v}^{10}$ ). Un valet arrive, vêtu d'une cotte si mautaillie et si detrenchie que a painnes en i avoit il la moitié de tele come ele avoit esté devant. Kex le surnomme d'emblée li Vallés a la cote Mautaillie : a tel eur dist Kex li senescaus ceste parole c'ainc puis ne li caï li nons. Le jeune homme explique qu'il a été attiré à la cour par la valeur d'Arthur, dont il fait l'éloge et à qui il demande de le faire chevalier. Le roi l'interroge sur son lignage, et le valet regrette qu'il veuille le faire chevalier "par lingnage et non mie par courtoisie ki en vous soit, ne pour bonté que vous esperés en moi » (f. 43v). Gaheriet et Lamorat rappellent à Arthur l'exemple de Lancelot, qu'il a fait chevalier sans rien savoir de lui : le roi accepte d'adouber le jeune homme. C'est donc le modèle de Lancelot qui se dégage, ce qui se confirme lorsque le jeune homme explique que son père a été tué (comme Ban) et que la cotte tailladée qu'il porte répond au vœu qu'il a fait : son vêtement est déchiré d'autant de coups que son père a reçus en trahison et il le portera jusqu'à ce qu'il l'ait vengé.

\footnotetext{
${ }^{8} \mathrm{Je}$ reprends là deux pages de mon introduction à l'édition du tome $\mathrm{V}$ du Tristan en prose cit. note 1.

${ }^{9}$ Dans l'analyse de Löseth, le cor se trouve au $\$ 47$ et l'apparition de Brunor au $§ 66$.

${ }^{10}$ Cet épisode se situe antérieurement à ce qui est raconté dans le volume I de l'édition Ph. Ménard. L'édition Curtis, fondée sur le manuscrit de Carpentras, donne une version différente ( $§ 635$, p. 215).
} 
Son premier exploit, peu après, est de tuer le lion de la cour qui s'est échappé : se Lancelos du Lac proprement l'avoit fait, si diroit on que che seroit grans hardemens (f. 44v). Le Chevalier a la Cotte Maltaillie est un nouveau Lancelot.

Peu après un autre modèle se dégage. Quand une demoiselle arrive à la cour, en quête d'un chevalier qui veuille accomplir l'aventure des Détroits de Sorelois, personne ne se porte volontaire : seul Brunor se manifeste, au grand dam de la jeune fille qui aurait souhaité un chevalier expérimenté. Brunor commence par abattre Daguenet, le fou du roi. Vêtu d'une cotte déchirée, verte, se heurtant à Daguenet, Brunor est une sorte de fou, qui tient de Perceval : orphelin de père comme lui, il arrive à la cour, réclame d'être fait chevalier et prend en charge une aventure qui semble le dépasser ; Kex dote Brunor d'un surnom moqueur, tout comme il raille Perceval. Brunor serait donc un Perceval mâtiné de Lancelot. La prégnance du modèle de Perceval explique une incohérence du manuscrit de Vienne dans lequel le jeune homme dit au roi qu'il se nomme Brunor (f. 44), ce qui n'empêche pas Arthur quand il s'en va de dire qu'il ne connaît pas son identité (f. 45). L'ignorance qu'affiche Arthur s'explique par le modèle de Perceval.

Tenant d'abord de Lancelot et de Perceval, Brunor va ensuite se couler dans un moule tristanien, tant est forte dans cet univers l'attraction qu'exerce l'amant d'Iseult. Sans cesse on le verra rivaliser avec Tristan, aimer Iseult sans espoir, chanter, se battre... Brunor, double de Lancelot, Perceval et in fine Tristan, illustre bien le dévoiement de la quête, du Graal à la dame, en particulier Iseult. En cela il est représentatif du Tristan en prose dont il me semble être une des grandes inventions. Qu'est donc cette cotte maltaillie qu'il portera durablement et à laquelle il doit son nom ?

\section{c. La cotte maltaillie}

Lorsque Brunor entre dans le roman, il est d'abord caractérisé par une cotte maltaillie : La u il aloient ensi parlant entr'aus de monsigneur Tristran et de Palamidés, atant es vous laiens venir.I. vallet grant et bien taillié, et d'aage ne pooit mie avoir plus de .XIX. ans. Li vallés vint si seus laiens $k$ 'il ne mena avoec lui ne escuier ne damoisele. Li vallés estoit mout bien cauciés a la guise de sa terre, mais de toutes robes il n'avoit que sa cemise et sa cote ki estoit d'un vert samit ouvré a or, mais ele estoit si mautaillie et si detrenchie que a painnes en $i$ avoit il la moitié de tele come ele avoit esté devant (f. $43 \mathrm{v}$ c). 
L'expression cote mautaillie figure dans le Conte $d u$ Graal: Perceval, chez Gornemant, abandonne sa cotel de cerf mal fete et mal taillie (v. 1420-1) ${ }^{11}$. La cotte de Brunor est verte, couleur de ce monde sauvage d'où vient aussi Perceval. Vers la fin du roman, Brunor circulera incognito et sera le Chevalier à l'Ecu Vermeil : peut-être devra-t-il alors la couleur de ses armes au Chevalier Vermeil du Conte du Graal. Quoi qu'il en soit, il n'est pas impossible que Brunor doive sa cotte à Perceval. Ce nom de Valet a la Cote Maltaillie attire l'attention et confirmerait dans un premier temps le modèle de Perceval, d'autant que l'état du vêtement est expliqué par le meurtre du père. Perceval, comme Brunor, est orphelin d'un père mort de mort violente. Par ailleurs, la scène de l'arrivée de Brunor à la cour présente des traits d'humour, qui ne sont pas sans rappeler ce qu'ont de comique les premiers pas dans la vie chevaleresque de Perceval. Ainsi on commence par nous dire que le jeune homme est grand et bien taillié alors que la cotte qui lui donnera son nom est mautaillie : l'opposition bien / mal taillié peut prêter à sourire. Le bref portrait amuse aussi, je crois, quand il mentionne la grande taille, l'âge et l'isolement du jeune homme que personne n'accompagne, avant d'enchaîner sur le fait qu'il est cauciés à la guise de sa terre (comme si on le dévisageait de la tête -sa grande taille- aux pieds, aux jambes) ${ }^{12}$. D'ailleurs c'est Kex qui le premier commente l'arrivée du garçon et qui lui donne son surnom : il donne le ton, celui de l'humour.

L'écho bien taillé / mautaillé attire tout de suite l'attention sur la cotte. Or l'adjectif mautaillé est énigmatique. On nous dit certes d'emblée que la cotte est tailladée en souvenir du meurtre du père. Pourtant cette explication n'est pas convaincante et elle semble n'être qu'une rationalisation superficielle d'un élément plus profond. En effet, jamais dans le Tristan en prose il ne sera question de la vengeance de ce père. Pourtant le roman est suffisamment long pour qu'un épisode de cet ordre ait pu y trouver place. Certes, nous ne sommes pas dans la Suite du Merlin post Vulgate où il n'est question que de pères, de frères ou de sœurs à venger, la vendetta n'est pas obsessionnelle dans le Tristan. Cet oubli n'en paraît pas moins surprenant : si le motif avait été essentiel, Brunor n'aurait pas manqué de venger son père. Or il n'en est rien, alors que le nom de Valet puis Chevalier à la Cotte Maltaillie lui colle à la peau et qu'il semble porter durablement ce vêtement.

Par ailleurs detaillé serait plus approprié que mautaillé pour décrire un vêtement tailladé par des coups d'épée : c'est d'ailleurs le terme qu'emploie le manuscrit de Vienne au

\footnotetext{
${ }^{11}$ Ed. F. Lecoy, Paris, Champion, 1973.

${ }^{12}$ On peut se demander si ces chausses a la guise de sa terre ne rappelle pas les chausses faites par la mère de Perceval (celui-ci porte des braies feites a la guise / de Gales, ou l'an fet ensanble / braies et chances, ce me sanble v. 498-500).
} 
folio 43v c. Lorsque Arthur voit le jeune homme, il commente : «Del vallet ne me sanble se bien non, fait li rois, mais de la cote k'il porte me pert mal, ki si malement est détaillie ». Mautaillie pourrait certes signifier que le vêtement n'est pas à la taille du valet (qui est grand), ce qui peut s'expliquer car il semble bien qu'il s'agisse de la cotte du père. Néanmoins on a l'impression qu'à la base il y a un nom, Valet à la Cote Mautaillie, qui aurait été rationalisé par detaillé, tailladé, d'où l'invention, finalement sans grand écho, du meurtre paternel : à la remarque d'Arthur qui s'étonne de la cotte detaillee, Kex répond en donnant le surnom de Valet a la cote Mautaillie, sans reprendre le terme du roi, comme cela aurait été logique dans ce type d'échange. Le surnom du personnage, surnom qui le prédétermine, à partir duquel il semble avoir été inventé et venir directement d'une cotte maltailliee préexistante, bien connue.

Quelle est cette cotte ? On trouve l'expression cotte maltaillie, nous l'avons vu, dans Le Conte du Graal de Chrétien, pour désigner le vêtement de sauvage -le pull tricoté par maman- auquel le jeune homme doit renoncer chez Gornemant. Le vêtement marquerait le lien à l'enfance (au père dans le Tristan, à la mère chez Chrétien). L'écho me paraît plausible. Cependant le parallèle entre l'arrivée à la cour de Brunor et celle du héros dans le lai du Mantel me semble aller aussi dans le sens d'un rapprochement entre la cotte maltaillie et le mantel mautaillé : dans le lai comme dans le Tristan, un jeune homme, de belle prestance, inconnu, arrive à la cour, sans compagnie, il ne connaît pas le roi, et c'est Kex qui doit le renseigner ${ }^{13}$. Le rapprochement a d'ailleurs pu être favorisé par une autre dénomination du lai, le Cort mantel ${ }^{14}$, cort ressemblant phonétiquement à cotte. Cette hypothèse paraîtrait fragile si rien ne venait l'étayer. Or elle me semble enrichir la conjointure du roman et expliquer l'importance accordée à Brunor, ce nouveau venu arthurien, qui jouera un rôle important à la fin de l'histoire, puisqu'il accompagnera Tristan à la Joyeuse Garde. A défaut de l'intentio auctoris, c'est au moins l'intentio operis qui se dessine là, pour reprendre la terminologie d'Umberto Ecco.

\section{d. Le cor et le mantel}

Un indice important étaie cette hypothèse. Le cor est associé à Lamorat, par qui s'opère le détournement vers la cour d'Arthur. Le mantel est associé à Brunor. Or ces deux

\footnotetext{
${ }^{13}$ Le lai du Cor et le Manteau Mal taillé. Les dessous de la Table Ronde, éd. et trad. N. Koble, Paris, Editions Rue d'Ulm, 2005, p. 58-59 (v. 108-ss).

${ }^{14}$ Voir éd. N. Koble, note a p. 54.
} 
personnages interviennent dans le manuscrit de Vienne à moins de trente folios d'intervalle. Or, comme Damien de Carné l'a montré dans sa thèse ${ }^{15}$, Lamorat et Brunor entretiennent un rapport d'équivalence, qui les amène à se succéder (dès le début, comme le montre le fait que Brunor arrive à la cour alors qu'il vient d'être question de Lamorat). Le rapport d'équivalence entre les deux personnages invite à associer le cor de Lamorat et le mantel de Brunor, tout comme sont liés thématiquement les deux lais, du cor et du mantel, centrés sur deux objets qui, traditionnellement, mettent à l'épreuve la chasteté ou la fidélité des femmes ${ }^{16}$. A une reprise explicite du lai du Cor, succèderait une allusion au lai du Mantel, masquée par la substitution d'une cotte au mantel attendu et par le fait que l'état du manteau est expliqué par une vendetta familiale.

Quel sens donner à cette configuration ?

Brunor aimera Iseult tout au long de sa vie, sans jamais renoncer, sans jamais fauter. Il incarne la soumission absolue à la Dame. Son amour fait de lui un double de Tristan: Lancelot combattant contre lui pensera affronter le neveu de Marc ; Brunor explique qu'il a les mêmes talents que Tristan, l'escremie et les échecs $(\$ 97)^{17}$; lui aussi compose des lais. Brunor cependant n'est pas une simple doublure. Le doublement sombre Brunor le Noir est plus qu'un double de Tristan $(\S 21,82)$ : c'est un Tristan superlatif, un Tristan qui n'est pas aimé, et qui ne meurt pas. Il incarne la fidélité amoureuse absolue, sans faille et sans retour. Il incarne aussi plus parfaitement que le neveu de Marc l'idéal chevaleresque : il croit à la droiture (dans le combat du Château Etroit, alors même que la lutte peut sembler inégale $\S 82,30, \S 86,69$-ss) ; lors du duel judiciaire, il est le seul à suivre les règles : il veille la nuit avant le combat, il prie, il se signe avant d'entrer sur le champ de bataille (§83). A la fin du roman, il semble presque dépasser un Tristan usé par neuf tomes de l'édition Philippe Ménard. Dans le château où une demoiselle chante le Lai de Recort, c'est Brunor et non Tristan qui est reconnu (§58-59). Brunor incarnerait l'idéal chevaleresque et amoureux plus

\footnotetext{
${ }^{15}$ Foisonnement et organisation. Réflexions sur la structure du Roman de Tristan en prose, thèse soutenue à Nancy 2 en 2005 sous la direction de B. Guidot.

${ }^{16}$ Voir par exemple E. Baumgartner, « A propos du Mantel Mautaillé », dans Romania, t. 96, 1975, p. $315-332$; P. E. Bennett, « Le lai du cort mantel et la critique de la courtoisie », dans Les Lettres romanes, t. 32, 1978, p. 103-121 ; H. Bloch, «The arthurian Fabliau and the Poetic of Virginity », dans Continuations. Mélanges J. L. Grigsby, éd. N. J. Lacy et G. Torrini-Roblin, Birmingham, 1989, p. 231-249; S. Hofer, « Bemerkungen zur Beurteilung des Horn et des Mantellai », dans Romanische Forschungen, t. 65, 1953, p. 38-48 et l'éd. cit. de N. Koble pour une bibliographie plus complète p. 177-178.

${ }^{17}$ Les références concernant le Tristan portent toutes désormais sur le t. V, éd. C. Ferlampin-Acher, citée note 1.
} 
efficacement que Tristan. Son amour impossible le laisse libre pour l'aventure et pour défendre les valeurs de la chevalerie. Contrairement à Palamède ou Celicès, il ne meurt pas : il est l'avenir du roman de chevalerie et Guiron le Courtois ne l'oubliera pas, qui lui inventera un père.

Brunor n'est donc peut-être pas qu'un double de Tristan. Quand, après le combat du Château Etroit, il commence à se dévoiler (on voit alors son visage désarmé $\$ 90,8-11$ ), il se met à chanter des lais, comme Tristan, mais il refuse de porter aucun escu des armes autrui $(\S 93,16)$ et ne chante que ses propres œuvres : cette formulation peut étonner, car justement il vient de porter les armes de Tristan lors du combat qui l'a opposé à Lancelot. Inconstance du personnage ? Sourire du narrateur? Certes, mais pas seulement. C'est en toute ignorance qu'il accepte les armes de Tristan, alors que c'est consciemment qu'il refuse de jouer les compositions de l'amant d'Iseult. Brunor n'est pas un autre Tristan, au sens qu'il n'en est pas un clone ; il est un Tristan autre, un Tristan qui vivra, mais qui n'est pas aimé, tant il est clair qu'on ne peut maintenir les amants en vie qu'au prix d'une trahison de leur histoire.

Brunor porte une cotte maltaillie: dans le lai, ce sont les dames infidèles qui sont affublées de ce vêtement, qui paraît bien déplacé dans le cas de Brunor. Pourtant, de même que l'épreuve du cor aboutissait narrativement à un escamotage de l'adultère puisque la cour décidait de faire comme si de rien n'était alors que sur le plan symbolique le discours d'Iseult ne faisait qu'amplifier sa faute personnelle, de même Brunor, sur le plan narratif, porte le chapeau (pardon, le manteau), il assume un vêtement qui ne lui va pas, mais sur le plan symbolique la faute d'Iseult, sa dame, se trouve exhibée à travers l'ensemble du roman. On aurait donc un double déni d'une sanction possible de l'adultère : la cotte, pas plus que le cor, ne conduira à une sanction de la faute, tout en la signalant ostensiblement. Le cor signale la faute, mais l'affaire est étouffée ; le mantel, à contretemps, est porté par l'homme le plus fidèle qui soit, mais il témoigne sur tous les chemins arthuriens qu'il y a faute. Le déni est double : il n'en faut pas moins pour que se prolongent les aventures de Tristan et Iseult. Le signalement est double : il n'en faut pas moins pour que meurent les deux amants.

Le cor ne donnerait pas lieu à un simple épisode, récriture du lai sur le mode compilatoire : il est un élément obvie, qui invite à relire la cotte, cryptée. La différence est donc nette avec l'épisode de la première Continuation, où l'épreuve du cor est remportée par $\operatorname{Caradoc}^{18}$ : en dépit de points communs ${ }^{19}$, une différence majeure réside dans le fait que le

\footnotetext{
${ }^{18}$ Ed. W. Roach, trad. C. A. Van Coolput-Storms, Paris, Le Livre de Poche, Lettres Gothiques, 1993, v. 31063271.
} 
récit centré sur le cor se prolonge dans le Tristan en prose. Dans le lai, l'aventure du cor est autonome, dans la Continuation, elle clôt la branche consacrée à Caradoc et permet de sortir définitivement Guinier du récit, tant il est vrai qu'une héroïne si parfaite ne peut pas intéresser le roman: dans le Tristan au contraire l'aventure continue au-delà. Le roman en prose banalise le succès et la perfection, puisque quatre dames accompliront l'aventure et resteront néanmoins anonymes, mais en même temps il signale l'inefficacité narrative et humaine de cette perfection.

Si dans le Tristan le cor est explicitement associé à la fidélité alors que le cotte travaille le texte plus souterrainement, dans Guillaume d'Angleterre, le cor et la cotte fonctionnent explicitement en binôme, tandis que la problématique de la fidélité, loin d'être explicite, doit être décryptée.

\section{Guillaume d'Angleterre}

Guillaume d'Angleterre est un roman en vers, racontant le destin du roi d'Angleterre, de sa femme Gratienne et de leurs deux fils, Lovel et Marin : le début du récit reprend fidèlement le modèle de la vie de saint Eustache, le couple se dépouillant de tous ses biens pour partir à l'appel de Dieu, la reine mettant au monde deux jumeaux dans la forêt, avant d'être enlevée par des marchands et séparée de Guillaume, les deux nouveau-nés étant adoptés par des bourgeois. Le dénouement, qui rend le trône au deux protagonistes et leur évite le martyre, s'écarte nettement de la trame hagiographique. Dans cette histoire, un cor joue un rôle essentiel : le roi, en fuyant, laisse dans sa chambre un cor qui est trouvé par un enfant (v. 412-418). Plus tard, devenu marchand, Guillaume rencontre à la foire de Bristol un jeune homme, celui-là même qui trouva le cor, qui lui vend ce souvenir (v. 2067-2119). Un jour, Guillaume arrive par bateau dans le pays dont Gratienne est devenue reine et elle reconnaît le cor, pendu au mât du navire (v. 2435-59). Elle se fait donner, au nom de la coutume qui l'autorise à prendre dans tous les bateaux l'objet qu'elle préfère, un anneau qui

\footnotetext{
${ }^{19}$ L'argument d'Iseult invoquant un enchantement dans le Tristan pour mettre en doute la fiabilité du cor est vraisemblablement inspiré de la ruse de Guenièvre qui cherche dans la Continuation à désamorcer l'épreuve d'abord en signalant le danger que représente un enchantement, puis en priant Dieu de faire échouer le roi s'il tente l'aventure malgré l'interdiction de son épouse (v. 3182-ss) ; le succès rapidement mentionné dans le roman en prose de quelques anonymes peut servir à intégrer les succès de Caradoc (dans le lai et dans la Continuation); l'escamotage final est commun au Tristan, au lai et à la Continuation.
} 
lui a appartenu et que Guillaume avait gardé. La reconnaissance se fera progressivement et à la fin le jeune garçon qui a trouvé le cor deviendra chambellan du roi. Quant à la cotte, c'est celle que portait le roi au moment de sa fuite : il la coupe en deux pour langer les nouveau-nés dans la forêt. Les bourgeois qui élèveront les deux garçons leur rendront plus tard ces langes et ces pans permettront la reconnaissance avec Guillaume.

Le cor et la cotte sont les deux seuls biens qui, distingués au milieu de l'ensemble des possessions royales, perdus et retrouvés, jouent le rôle de signes de reconnaissance. La mise en relation des deux objets est claire.

Dans Guillaume d'Angleterre, le cor est polysémique. D'une part, il est la marque du pouvoir royal, perdu et retrouvé, comme le montre A. Magnusdottir ${ }^{20}$. D'autre part il renvoie à la passion du roi pour la chasse : le modèle narratif est la vie de saint Eustache ; le roi, invité par Gratienne après leurs retrouvailles, a une hallucination cynégétique, qui pousse la dame à organiser pour lui une chasse, au cours de laquelle il retrouve ses fils. Au début du roman, renonçant à sa condition, le roi abandonne la chasse, laisse son cor : il reconquerra peu à peu ce privilège, ses fils manifestant d'ailleurs, en dépit d'une éducation bourgeoise, d'étonnantes aptitude à la vénerie (par exemple lorsque Lovel tue un jeune daim dans la forêt du seigneur de Catenasse v. 1737-ss).

La cotte que le roi coupe en deux pour couvrir les deux nouveau-nés que la reine vient de mettre au monde dans la forêt renvoie certes au modèle hagiographique (à saint Martin ou saint Gilles) mais les pans conservés par les bourgeois qui ont élevé les enfants permettent in fine la reconnaissance et le retour au modèle familial et féodal, avec éviction du martyre. Le texte, réservant le cor à la relation entre la reine et le roi, et la cotte coupée en deux à la relation entre les fils et le père, répartit les rôles sur le plan narratif et invite, sans aucune ambiguïté, à les rapprocher : dans les deux cas, à partir d'un modèle hagiographique, on glisse vers une restauration féodale. Autant la mise en relation de la cotte et du cor demandait à être reconstruite dans le Tristan, autant elle est évidente ici, les deux objets étant placés sur le même plan. En revanche, si le rapport au cor servant de test de fidélité est explicite dans le Tristan, il est nettement plus crypté dans Guillaume.

Pourtant il se laisse découvrir à partir d'un indice surprenant. Le cor est sous le lit lorsque le jeune garçon le trouve (v. 413). C'est une place bien étrange, et d'ailleurs le

\footnotetext{
${ }^{20}$ La voix du cor : la relique de Roncevaux et l'origine d'un motif dans la littérature du Moyen Age XIIe-XIVe siècles, Amsterdam, Rodopi, 1998, p. 93-ss.
} 
manuscrit de Paris (BnF. fr. 375) au vers 2100, dans le récit rétrospectif que le garçon fera de la scène lors de la foire de Bristol, mentionne qu'il a été trouvé sur un banc, et non sous un lit (le manuscrit de Cambridge ne commet pas cette erreur). Comment interpréter cette contradiction? Par le souci du jeune homme de rendre vraisemblable la scène, un cor se trouvant plus facilement sur un banc que sous un lit? A moins que le jeune garçon n'ait perçu ce qu'il peut y avoir d'inconvenant à mettre un cor sous un lit et qu'il ait remplacé le lit par un banc, plus décent. En fait, la localisation du cor sous le lit me parait essentielle. Le cor renvoie en effet au plaisir de la chair : le roi, partant en exil, perdant sa femme, renonce à ce plaisir, le cor (phallique ou homonyme du corps) restant sous le lit.

A la lumière de cet indice, la «faute " du roi et celle de la reine, fautes qui les poussent à l'exil, trouvent un nouvel éclairage. Le portrait qui est donné d'eux au début du roman ne laisse a priori deviner aucune faille : il ne signale que leur perfection et leur amour absolu. Mais après qu'une manifestation céleste a incité trois fois le roi à partir en exil et à abandonner tous ses biens, le chapelain invite son seigneur au repentir, et le portrait idéal se fissure quelque peu, sans que pour autant la faute soit claire :

«Mais je sais bien que vos avés

Mainte cose u vos n'avés droit.

Faites crïer tost orendroit,

Se nus vos set que demander,

Car pres estes de l'amender» (v. 97-100).

Le roi se hâte de rendre son bien à chacun (v. 108-ss) : il convoque sans tarder Trestous ciax de cui il savoit / Que riens du leur a tort avoit (v. 109-110).

Sa faute à ce stade paraît être la cupidité, qu'il expiera en se dépouillant de tout ${ }^{21}$. L'épisode de l'aumônière (v. 723-ss) constitue le point culminant de ce dépouillement : après avoir refusé les cinq pièces d'or données par le marchand qui emporte sa femme, le roi perd ses deux fils et décide de retourner prendre la bourse, qu'un aigle, la voyant rouge comme de la viande, lui arrache au moment même où il allait mettre la main dessus (v. 877-ss) (on reconnaît là un motif que l'on retrouve dans l'Escoufle). Le roi prononce alors un vif réquisitoire contre Convoitise (v. 886-924) et renonce chrétiennement aux biens matériels : plus tard, il rachète le cor au jeune garçon, non pour sa valeur matérielle, mais pour ce qu'il représente affectivement (v. 2067-ss). Il est passé du matériel au symbolique. Quant à

\footnotetext{
${ }^{21}$ Les réflexions qui suivent sur la faute sont empruntées à mon introduction à l'édition cit. note 1.
} 
l'aumônière, c'est un miracle qui la lui rend (v. 2825-ss) : Diex la lui envoia en presant ; ses fils voient dans cette aventure un miracle indéniable (v. 2834-2836) ${ }^{22}$.

Pourtant, Guillaume devient commerçant, fait ses preuves, remonte sur le trône et prend pour conseiller un bourgeois. Les valeurs marchantes semblent mises à l'honneur. Cette restauration inverse complètement le mouvement initial et invite à revenir sur la conversion du roi, d'autant que dès le début le chapelain promet à Guillaume que :

«(Et) Diex, quant li termes venra,

A.C. doubles le vos rendra:

Ne descroistra pas vostre moebles,

Car vos rarés tot a.C. doubles,

Le guerredon et le merite ». (v. 161-165)

Le dernier vers, qui peut laisser croire à une conversion des biens terrestres en bénéfices spirituels, est invalidé par le vers 163 où il est bien question de richesses matérielles. Par ailleurs le thème de l'argent est récurrent dans le texte : le va-et-vient permanent entre le matériel et le spirituel n'implique pas une conversion de l'un en l'autre, mais au contraire une sorte de confusion prosaïque, qui brise tout élan rédempteur. A la fin du roman, il n'est guère question de salut, alors que les mentions de la valeur des rentes attribuées aux uns et aux autres envahissent le texte (v. 3323, v. 3325). Selon moi, c'est à une hiérarchisation que le lecteur est invité : le temporel et le spirituel ne doivent pas être confondus : il faut rendre à César ce qui est à César. Le roi, même s'il aspire au martyre, même si son autorité est à la fois spirituelle et temporelle, exerce son pouvoir dans le siècle, comme les marchands. Un roi qui s'exile et joue à saint Eustache, et c'est le royaume qui est menacé, comme le montre le pillage que subit le palais au départ de Guillaume. Dès lors, on comprend la mise à l'écart que subit la chevalerie dans ce roman, dans la mesure où elle mêle dans ses pratiques et ses motivations le temporel et le spirituel, comme en témoigne à l'époque l'évolution de l'adoubement: Roland, qui sert de comparant à Gliolas, désormais vieux et décrépit, est renvoyé à un passé révolu (v. 1055) ; les guerres des barons, auxquels sont comparés les vents

\footnotetext{
${ }^{22}$ Pour une lecture chrétienne et providentielle de cet épisode, voir F. Danelon, «Sull'ispirazione e sull' autore del Guillaume d'Angleterre », dans Cultura Neolatina, t. 11, 1951, p. 49-67 et S. Sturm-Maddox, "Si m'est jugie et destinee : on Guillaume d'Angleterre », dans The Sower and his Seed : Essays on Chrétien de Troyes, éd. R. T. Pickens, Lexington, French Forum, 1983, p. 66-80.
} 
tempétueux, sont dénoncées dans un passage hautement rhétorique (v. 2330-2342) ${ }^{23}$. La faute du roi n'est donc pas l'appât du gain.

En fait, plus que l'argent, c'est une cupiditas très large qui est en jeu : elle concerne le roi, mais aussi et surtout la reine. Comme le montre Francine Mora de façon très convaincante, la réflexion sur la convoitise n'est pas purement chrétienne : la référence à Tantale, dans le monologue que prononce le roi après que l'aumônière lui a échappé (v. 903) Tantale que le dit du XIVe siècle, plus édifiant, remplacera par $\mathrm{Job}^{24}$-, le prouve clairement. Derrière cette cupiditas, c'est le désir, l'appétit de chair, qui est au cœur du roman. Appétit de chair que le roman prend souvent au sens littéral (d'où la fringale de Gratienne qui vient de mettre ses deux enfants au monde v. 545-ss ou l'importance accordée au repas préparé par Marin, Lovel et Rodain devant la loge du forestier v. 1764-ss), mais qui au fond est sexuel :«l'appétit de chair est très ambigu», et renvoie aux pulsions sexuelles, la faim de Gratienne étant métaphorique d'un désir incestueux, comme dans Apollonius de Tyr, «l'un des modèles probables de notre conte $»^{25}$. La reine refuse la cuisse que lui propose son mari : elle rejette la chair qu'il serait normal qu'elle accepte, celle de son époux ${ }^{26}$, et préfère celle, interdite, de ses enfants.

La faute initiale est donc imputable autant à Gratienne qu'à Guillaume. Cette hypothèse est d'autant plus vraisemblable qu'on peut s'interroger sur la naissance des jumeaux. Le couple attend cinq ans sans avoir d'enfant. Le motif est banal et annonce souvent la naissance d'un héros. Ici cependant ce sont deux héros qui viennent au monde. Cet élément est certes inspiré directement par la vie de saint Eustache. Cependant il me semble qu'il est relu dans une perspective qui n'a rien d'hagiographique. Le lai de Fresne de Marie de France se fait en effet l'écho d'une croyance imputant à la mère de jumeaux des relations avec deux

\footnotetext{
${ }^{23}$ Voir sur ces points K. Holzmayr, « La métamorphose du roi Guillaume », dans Médiévales, t. 4, 1983, p. 91 101.

${ }^{24}$ " La fabula antique comme matrice des premières mises en roman : l'exemple de Guillaume d'Angleterre », art. cit., p. 296-ss.

${ }^{25}$ Ibid., p. 301. L'énigme proposée dans ce roman, maternam carnem vescor ( « je me repais de la chair de ma mère ») renvoie à l'inceste et correspond au même schéma, quoique inversé, que Guillaume d'Angleterre. Tout cela est contre nature, ne serait-ce que parce que la reine risque d'être enceinte à nouveau des enfants qu'elle a déjà mis au monde (v. 616-617).

${ }^{26}$ Avec son second mari, cette fois-ci pour la bonne cause, il en ira de même, ce qui n'est pas sans ambiguïté.
} 
pères $^{27}$. Certes Fresne dénonce cette croyance. Mais la tentation incestueuse que nous avons pu deviner à la suite de F. Mora dans la scène de l'accouchement nous oriente vers une reine inquiétante : l'hypothèse d'une Gratienne adultère, hypothèse que je propose avec prudence, confirmerait le déplacement de la faute du roi vers la reine, les jumeaux sanctionnant le péché maternel. Le désir de la reine se serait détourné de son mari vers un amant, puis vers ses fils : la suite du roman raconterait la renaissance du désir au sein du couple royal, par le biais de deux objets hautement symboliques, l'anneau et le cor.

Dans ce contexte, la chasse, qui joue un rôle très important dans ce récit qui s'inspire de la vie de saint Eustache ${ }^{28}$, pourrait être une métaphore du désir sexuel, comme le suggèrent l'ambiguïté du terme deduit qui désigne à la fois le plaisir amoureux et la chasse (v. 2569), et le fait que la chasse que le roi préfère est celle du cerf en rut (v. 2570). D'ailleurs Guillaume, alors qu'il vient de reconnaître sa femme, a un fantasme, non pas érotique, mais cynégétique (v. 2568-ss). Voyant des chiens, il se met à rêver éveillé et à penser à la chasse qu'il aimait tant : sa femme organise alors pour lui une sortie en forêt. Le lecteur aura peut-être à l'esprit la chasse de Didon et Enée dans Eneas, mais on notera qu'ici la reine reste en arrière, ne suit pas le roi qui sera seul lors de la rencontre avec les deux fils (ce qui, si l'on suit l'hypothèse d'un désir incestueux chez Gratienne, revient à mettre définitivement celui-ci à l'écart puisque la reine est restée, volontairement, éloignée ${ }^{29}$ ). Dans cette perspective, on comprend que le cor n'est pas qu'un objet secondaire : il est certes le symbole des vanités auxquelles le roi renonce et du pouvoir royal abandonné puis reconquis, mais il me semble qu'il faut aussi lui donner une symbolique sexuelle puissante, en relation avec le désir du roi. Dans cette perspective, la scène du cor trouvé sous le lit doit être relue en relation avec le cor associé à l'épreuve de chasteté que l'on rencontre dans le Lai du Cor ou dans les aventures de Caradoc dans la Première Continuation du Conte du Graal $^{30}$. Si l'on accepte l'hypothèse proposée à partir du

\footnotetext{
${ }^{27}$ Lais, éd. J. Rychner, Paris, Champion, v. 38-42. Le heurt entre l'hagiographie, où les saints ont souvent des jumeaux (pensons à Gervais et Protais, les jumeaux engendrés par saint Vital et sainte Valérie), et la superstition mentionnée par le lai est violent, et concourt à l'ambiguïté du roman.

${ }^{28}$ H. Klüppelholz, «Zur Deutung der Jagdepisoden im Guillaume d'Angleterre», dans Archiv für das Studium der neueren Sprachen und Literaturen, t. 227, 1990, p. 298-305.

${ }^{29}$ La mise à l'écart du modèle oedipien, bien connu depuis le Roman de Thèbes, est confirmée par le fait que les fils ne tueront pas le père et que les identités seront clarifiées sans tarder.

${ }^{30}$ Voir N. Koble, Le Lai du Cor et Le Manteau mal taillé. Les dessous de la Table Ronde, cit. note 12, en particulier l'introduction p. 103-ss et E. Baumgartner, "Caradoc ou la séduction», dans Mélanges Alice Planche, Annales de la faculté des Lettres et Sciences Humaines de Nice, Paris, Les Belles Lettres, 1984, p. 61 69.
} 
lai de Fresne selon laquelle la reine n'a pas été fidèle au roi, le cor aurait été abandonné sous le lit après avoir révélé la faute de la reine. La scène du cor a eu lieu avant le roman, elle reste cryptée, scandaleusement indicible.

L'épisode où le roi rachète le cor (à un jeune homme bien pieux qui veut aller en pèlerinage à saint Gilles) illustrerait le pardon du roi ; celle où Gratienne admire le cor et son mari avec les yeux de l'amour marquerait la fin du dévoiement du désir de la reine, désir égaré vers un amant, vers ses fils, et enfin revenu à son mari. Cette lecture n'est certes qu'une hypothèse, mais elle est appuyée par une tradition bien attestée, et elle permet de mettre en évidence une logique globale à un texte dont on a souvent critiqué l'incohérence.

Quelle est alors la place de la cotte ? Le roi coupe celle-ci en deux pour couvrir les jumeaux nouveau-nés, qu'il identifiera ensuite grâce à ce linge. Le motif est banal : il suffit de penser au lai de Fresne F $^{31}$

De riens ne vaurent samblant faire

Tant qu'il orent les pans veüs.

Li rois les a bien reconnus,

Et dist por voir que ce sont il.

Lors li font joie andoi si fil. (v. 2884-2888)

Ce qui constitue Guillaume en père, c'est d'une part le souvenir de la séparation et d'autre part le don qu'il a fait de son vêtement. A aucun moment il n'est question du sang qui parle, de reconnaissance immédiate... La famille se reconstitue grâce aux récits que font les enfants et grâce au tissu, que l'on peut interpréter comme une métaphore du texte : les retrouvailles tiennent avant tout à la parole. Si le texte laisse en suspens le problème de l'héritage de Guillaume et ne conclut pas sur sa lignée, c'est bien que le problème du sang était insoluble et que l'aborder risquait d'aller à l'encontre du rapprochement des deux frères qui oriente le roman. Finalement, les soupçons d'adultère et d'inceste qui planent sur la mère, concentrés au moment de la naissance des jumeaux, particulièrement étonnant, sont désamorcés (en partie) par les retrouvailles et la restauration qui terminent le roman. Le tissu, coupé et reconnu, servira de masque à la faute.

La conjonction du cor et de la cotte renverrait à la faute première, à une infidélité de la reine, qui aurait raté le test du cor. Le cor aurait été abandonné sous le lit. La cotte coupée en

\footnotetext{
${ }^{31}$ L'influence de Fresne sur Guillaume d'Angleterre me semble probable: dans les deux cas naissent des jumeaux (ou des jumelles), la reconnaissance finale se fait grâce à un tissu, les enfants portent des noms en relation avec des éléments naturels (arbres, animal, mer).
} 
deux pour langer les fils concrétiserait la faute et la rappellerait, car si la cotte est coupée c'est qu'il y a eu deux enfants. Comme dans Tristan la conjonction du cor et de la cotte déchirée marque la faute, le péché de chair, l'infidélité, le cor en étant l'épreuve ponctuelle, la cotte le symbole qui perdure. Si dans Tristan la faute d'Iseult est ainsi à la fois détournée et amplifiée, dans Guillaume d'Angleterre le cor est racheté et le tissu retrouvé. Mais l'on ne peut que douter de l'authenticité de la conversion, dans ce roman où tout rentre dans l'ordre premier. La meilleure solution que trouve le roi, c'est de faire du jeune homme qui a trouvé le cor son chambellan : il évitera ainsi que ce cor ou un autre ne traîne sous les lits. La solution, c'est effectivement le silence, le masquage, et ce n'est peut-être par pour rien que le roi, chez le bourgeois au service duquel il est entré, est devenu expert en teintures. Dans ce récit où il est question à plusieurs reprises du commerce de matières tinctoriales (v. 1985, v. 2193, v. 22442246), où le roi prend un pseudonyme (v. 998) et où la reine s'invente un passé de garce (v. 1144-ss), l'un et l'autre s'abaissant pour mieux reconquérir le pouvoir, ni teinture, qui donne de la valeur aux tissus (v. 2246), ni le mensonge, ni la fable, ne sont dévalorisés: le bourgeois à qui Guillaume ment, le croit, sur la foi de son apparence, tandis que les marchands à qui il dit une vérité peu en accord avec son allure lui refusent toute crédibilité (v. 642-ss). De là une mise à l'écart de la véridicité dans le monde des hommes : prime la ruse, la guille dont Guillaume porte le nom. Le tissu l'a emporté sur le cor.

Dans Guillaume d'Angleterre comme dans le Tristan en prose, on peut dégager une conjonction entre le cor et la cotte, posant le problème de la fidélité. D'un côté un objet remontant à un motif mythologique, associé à un processus narratif, une épreuve, de l'autre un symbole féodal. Des objets donc disparates, qui néanmoins réussissent à former une configuration signifiante. Dans le Tristan, le rapport entre les deux est implicite et demande à être dégagé : la fidélité est en revanche mise explicitement en relation avec le cor. La conjonction des deux permet à la fois un double déni de la faute d'Iseult et un rappel insistant de celle-ci. Dans Guillaume, la configuration est plus évidente, mais sa senefiance est très ambiguë, le désir, l'appétit et la chasse, qui sont certainement les causes mêmes de la faute, amenant en même temps et paradoxalement la reconstruction du couple royal, tandis que le tissu, garant de la fidélité lignagère, se pare de teinture.

La proximité de ces deux objets, que l'on perçoit dans la parenté thématique et formelle des lais du Cor et du Mantel, est-elle le fait du hasard? Ou bien faut-il y voir un rapprochement dialectique entre le tissu, qui cache (mal parfois)le corps, et le cor qui signale et dénonce les fautes de ce même corps ? Dans le Tristan et Guillaume le cor révèle la faute ; 
le tissu dans le premier la proclame tout en la détournant, dans le second la masque. Quoi qu'il en soit, il me semble que nous avons là une conjonction dont on pourrait trouver d'autres attestations. Dans la première Continuation de Perceval $^{32}$, les aventures de Caradoc et Guinier se terminent par une épreuve du cor victorieuse. Juste avant le chevalier a recommandé à la demoiselle de se ceindre la poitrine d'une bande d'étoffe pour que personne ne puisse voir son sein en or. On a dans ce cas une étoffe qui garantit le secret du corps et un cor qui révèle que le secret a été gardé. Dans cette occurrence, l'ordre de la configuration est inversé, le tissu précède le cor : au lieu que la configuration soit dynamique et ouvre le récit, l'oriente et l'organise comme c'est le cas dans Guillaume d'Angleterre et Tristan, elle conclut les aventures Caradoc et Guinier, qui disparaît définitivement du texte. D'autres pistes mériteraient d'être étudiées : Aubéron, le nain bossu de Huon, n'est-il l'heureux possesseur d'un cor et d'un haubert (une cotte de maille) que nul, s'il n'est pas chaste, ne doit porter? Plus largement, sur le plan de l'imaginaire, la conjonction entre le cor et la cotte ne serait-elle pas à mettre en relation avec l'opposition entre le cor et la harpe ${ }^{33}$, le souffle insaisissable et la fibre (du textile ou, métaphoriquement, de la corde), entre la voix et le tissu, la parole et le texte?

\section{Résumé}

Guillaume d'Angleterre et le Roman de Tristan en prose n'ont en apparence guère de points communs. Cependant ces textes présentent tous deux une utilisation intéressante du cor, en relation d'une part avec l'histoire d'un couple marié, et d'autre part avec une cotte coupée, tailladée, celle du Chevalier à la Cotte Maltaillie dans le texte en prose, et celle que Guillaume dans le roman en vers partage en deux pour envelopper ses fils nouveau-nés. La ressemblance n'est-elle que superficielle? Ou bien peut-on mettre en évidence une configuration d'objets signifiante ? Dans les deux cas le texte travaille à partir du cor qui met la fidélité à l'épreuve, tel qu'on le trouve dans le Lai du Cor de Robert Biket. La reprise du lai du Cor est évidente dans le Tristan en prose et contribue à structurer fortement le roman, dans la mesure où elle trouve un répondant dans la figure de Brunor, le Chevalier à la Cotte Maltaillie, un double de Tristan. Dans Guillaume d'Angleterre, si la reprise du lai du cor n'est

\footnotetext{
${ }^{32}$ Ed. cit., v. 3106-3271.

33 Sur cette opposition, voir dans ce même volume mon article «L'essoufflement du cor dans la littérature arthurienne », p. 15 et 16.
} 
en rien explicite, la conjonction avec la cotte est obvie, puisque le cor et la cotte sont les deux attributs nobiliaires principaux dont se dépouille le roi. L'étude de ces deux textes montre que la conjonction entre les deux objets sert une réflexion sur la fidélité, le désir, la mise à l'épreuve du corps et son masquage.

Bio

Christine Ferlampin-Acher est professeur de langue et littérature médiévales. Elle travaille sur le roman, en particulier le roman arthurien (Perceforest, Artus de Bretagne). Elle a publié Fées, bestes et luitons. Croyances et merveilles dans les romans français en prose (XIIIe-XIVe siècles) (Presses Universitaires Paris Sorbonne, 2002) et Merveilles et topique merveilleuse dans les romans médiévaux ( Paris, Champion, 2003). A paraître en 2007 chez Champion Guillaume d'Angleterre (Champion Classiques) et Tristan en prose, BnF. Fr. 757, t. V (Classiques Français du Moyen Âge). 